\title{
Interactive comment on "Presentation and evaluation of the Arctic sea ice forecasting system neXtSIM-F" by Timothy Williams et al.
}

Anonymous Referee \#2

Received and published: 30 August 2019

In this paper the authors introduce a new sea ice forecasting system neXtSIM-F based on the neXtSIM sea ice model and present an evaluation of the model over a single season - winter 2018-19.

I feel that this study will be worth publishing in The Cryosphere (although it would likely fit better in GMD than TC). However several changes will be required before this is possible.

*General comments*

1. It is not made clear enough what the various runs and systems are that are assessed in this study. In particular there is also no mention of the "free run" before it is evaluated in Section 5.1. Section 3 contains information on the observational datasets used 
in this study but there is no equivalent for the model datasets. This study needs a summary of exactly which runs and systems are being evaluated with perhaps a table.

2. Additionally the names neXtSIM and neXtSIM-F seem to be fairly inter-changeable in the manuscript. I guess the neXtSIM-F forecasting system uses the neXtSIM sea ice model. If so then I think the name of your forecasting system as neXtSIM-F is a bit confusing.

3. The evaluation period is only a few months and does not include the late spring/summer period when many sea ice forecasting systems report their poorest performance. This means that it is hard to put the evaluation here into context with other operational systems. The conclusions of this study would be much strengthened if the authors could perform, and evaluate, a complete annual cycle (or preferably 2).

4. In general I find that there are too many figures and stats in the paper, which makes it hard to understand what the take-home message is.

*Specific comments*

1. In many places the language used in the paper is too informal and colloquial (i.e., in the abstract we have ". . . in our system, we obtain. .." and on P9 we have "the observed ones").

2. You need to be careful to distinguish between "sea ice concentration", which ranges from 0 to $100 \%$, and "sea ice area fraction", which ranges from 0 to 1 throughout this manuscript. For example in Figure 4 the caption says "concentration" but the scale is $+/-0.5 \%$. This is either a low "concentration" or a high "area fraction". I assumed the former to start with until I noticed that the text talks about an associated reduction in extent. With changes of $+/-0.5 \%$ concentration I wouldn't expect to see any departure to the $15 \%$ contour (extent) so is it actually "area fraction" plotted here?

3. I find the abstract to be rather technical and not very abstract. It reads a bit more like a conclusions/summary section. I would encourage the authors to make the abstract 
more exciting to make the paper more inviting to potential readers.

4. The introduction section (section \#1) is rather disjointed. It starts with some motivation for sea ice forecasting (with background on changing climate) but then jumps straight in to say that neXtSIM-F is based on neXtSIM. It doesn't actually say that neXtSIM(-F) is a sea ice forecasting system! It would be better to include a couple of extra lines to say that this is the case. Perhaps to say something like "Here we introduce a new sea ice forecasting system, neXtSIM-F, that is based upon the neXtSIM model....".

5. I find the introduction to operational ocean forecasting systems in Section 2 to be, almost paradoxically, both too detailed and non-existent. I say too detailed because I am left wondering why there is such a thorough introduction provided to the GOFS system when it isn't really used in this study? Of course, GOFS is only one of many global operational ocean-sea ice forecasting systems and you don't mention any others apart from TOPAZ and neXtSIM. The Tonani et al. (2015) GODAE paper provides a nice reference describing the world's operational global forecasting systems. Although several of the systems have doubtlessly moved on since 2015 , this reference provides evidence for the breadth of activity in the world. Tonani, M., Balmaseda, M., Bertino, L., Blockley, E. W., Brassington, G., Davidson, F., Drillet, Y., Hogan, P., Kuragano, T., Lee, T., Mehra, A., Paranathara, F., Tanajura, C. A. S. and Wang, H.: Status and future of global and regional ocean prediction systems, J. Oper. Oceanogr., 8, sup2, s201-s220, doi:10.1080/1755876X.2015.1049892, 2015.

7. The data sources section (\#3) does not make it clear which datasets are used for assimilation and which are used for evaluation (and hence which are used for both). At the least it is important to note which datasets are independent from the assimilation.

8. Related to the above point I find the description of the blended SSMIS+AMSR2 product somewhat confusing. Is this done purely for the evaluation? If not why can't the DA do this blending by waiting the observations with their respective errors?

Printer-friendly version

Discussion paper 
9. P3, L4: “. . profiles from Argo floats.”. Why do you only use Argo floats (if that's true)? Why not CTD/XBT/seals etc.?

10. I do not understand why a couple of weeks of CFSv2 is used in place or ECMWF. Surely you could get the replacement data from somewhere else (like ECMWF themselves for example)? If not then you should really consider the implications of using CFSv2. Specifically: is this the configuration with unrealistic ice growth caused by the fact that they turned off the stratus cloud formation to improve tropical temperatures and ENSO predictability (as described by Yang et al. 2017 and references therein)?: Yang, Q., M. Wang, J.E. Overland, W. Wang, and T.W. Collow, 2017: Impact of Model Physics on Seasonal Forecasts of Surface Air Temperature in the Arctic. Mon. Wea. Rev., 145, 773-782, https://doi.org/10.1175/MWR-D-16-0272.1

11. I don't like your "RMSE" for extent as it is exactly the Integrated Ice Edge Error (IIEE) of Goessling et al., (2016). You cite the ensemble extension of the IIEE (the SPS paper of Goessling and Jung, 2018) and say that your RMSE is like a deterministic version of that, which is misleading. It would be better to just cite the 2016 paper instead and call your metric "IIEE" instead of "RMSE": Goessling, H. F., Tietsche, S., Day, J. J., Hawkins, E., and Jung, T.: Predictability of the Arctic sea ice edge, Geophys. Res. Lett., 43, 1642-1650, https://doi.org/10.1002/2015GL067232, 2016

12. In Figure 3 I note that the neXtSIM concentration evolution is very smooth - more so than the low resolution SSMIS data - which I didn't expect given the resolution of the model. Can you comment on this? Is this caused by the fact that neXtSIM still uses the continuum formulation and so doesn't resolve small scale features?

13. I note with interest that MOSAiC forecasting is mentioned as a motivation for improving sea ice forecasts. There is an international project (SIDFEx) currently coordinating operational sea ice drift forecasts specifically to provide guidance to the Polarstern/MOSAiC. Presently the list of models includes TOPAZ but not neXtSIM. Are there any plans to contribute neXtSIM drift forecasts to SIDFEx? This might be an

Printer-friendly version

Discussion paper
Interactive

comment

\section{4}


interesting way to show the skill of neXtSIM in this regard.

14. Some of the figures (e.g., Figs 10\&11) suggest that the data assimilation is having a rather modest impact on the forecasts compared with many of the operational systems that I have seen in the past. Can you comment as to why that might be?

15. On page 20 it is mentioned that the "RMSE for drifters placed on the first day..." but this is the 1st time in the manuscript that drifters are mentioned. Can you explain this a bit more please?

${ }^{*}$ Figures*

As mentioned above I feel that there are too many figures in this manuscript. In particular in Figs 11 \& 12 there are 12 panels and each row looks virtually identical. Apart from telling me that the assimilation is having a rather modest impact, I don't understand what l'm supposed to do with all this information. Additionally the next similar set of figures, Figs 13-14, don't even seem to be discussed in the text at all. So are they necessary?

Many of the figure captions are too brief and should be improved. I believe that the Copernicus journal guidelines are that figures should be able to work stand-alone from the text, for which a bit more information is required.

I find that the $x$-axis date tick-marks provided on the time-series plots (Figs 3, 5, 7, 12 - less so for Fig 9) are not very useful. With such a short run period it would be better to include more dates. At the very least there minor tick marks should be used to show each day (or 5-days or something). It would also be good to specify this in the figure caption perhaps.

Figure 1 is a bit confusing because I am left wondering whether different time-scales are involved here. Is this a daily schematic or does it depict the whole run? For example the 2 top boxes (initialization) are surely not done each day are they? If not then it should be made clear what is done each day and what isn't - either in the caption or 
the figure (or both). Perhaps the initialization boxes could be enclosed in a dotted box or something?

I suggest you should also re-think your use of red-blue colour maps for sea ice concentration. I have seen people use red for less ice (as it's hotter) and blue for more ice (colder) in the past as well as the other way around. It might be better to avoid the use of a hot<->cold colour-map therefore.

*Technical corrections*

The 1st instance of "SSMIS" is correct but thereafter it has been changed to "SMMIS".

Also "first day (4th day)" appears in many places, which is not very consistent

P2, L26: CMEMS should be "Copernicus Marine Environment Monitoring Service"

P2, L29: ". . the version 4.1 of the..." - suggest to remove the 1st instance of "the" here

P2, L30: The reference for CICE v4.1 is Hunke and Lipscomb (2010): Hunke, E. C. and Lipscomb, W. H.: CICE: the Los Alamos sea ice model. Documentation and software users manual, Version 4.1 (LA-CC-06-012), T-3 Fluid Dynamics Group, Los Alamos National Laboratory, Los Alamos, 2010

P3, L30: "As specified by the validation reports above..." should be "As specified by the validation reports cited above..."

P4, L5: "metrics" should be "metric"

P4, L6: is extent "above $15 \%$ " or "at least $15 \%$ "? I thought the latter.

P4, L13: "...can be obtained for 48 hours..." sounds like only 48 hours of data. Do you mean this or do you mean the data is available 48-hours behind real-time?

P5, L5: calculating volume for each model \& obs based on thickness like this will involve different areas of ice won't it? 
P5, L26: "Modelling" is spelt incorrectly as "Modeling" in the NEMO acronym

P5, L31: “. . if the temperature is below 0C". Which temperature - surface skin temperature, or near-surface atmosphere temperature (T2M)? Please be more specific.

P10, L29: "of these variables" is not adding to this sentence and should be removed

P11, L6: ". . predicts the no ice...." Please remove the "the".

P11, L11: "averages values" should be "average values"

P11, L19: "underestimation in the Bering Sea". I presume that you mean the Chukchi Sea here because the Bering Sea is outside your model domain?

P13, L33-4: "land-fast" should be "land-fast ice"?

P13, L35: (as above) I suspect that "Bering Sea" should be "Chukchi Sea" here

P14, L9: the title "Evaluation of forecasts with assimilation" is confusing because I doubt that you are actually doing assimilation in your forecasts are you? Perhaps this should be changed to something more like "Forecasts performed from analysed ice conditions"?

P16, L9: Do you mean "significantly" here in the scientific sense of the word? If so include a p-value, if not I suggest changing to "considerably".

P18, Fig 9 caption: "(blue)", "(orange)", and "(red)" are provided but not "(green)"

P21, Fig 12 caption: "error bars" should be "shading"

P26, L7: "limited resources" suggests a deficiency in resource. You should change to "minimal resources" if you wish to suggest that the model is cheap to run.

P26, L20-21: “. . forecasts used saved atmospheric and ocean forecasts as forcing. ..”. What does this mean? 